\title{
Research progress on the safety evaluation of selenium enriched yeast
}

\author{
Benjiao $\mathrm{Li}^{1}{ }^{1,}$, Song $\mathrm{Li}^{1}$, Binrong $\mathrm{Ma}^{1}$, Yong Jing ${ }^{1}$, Zheming Zhang ${ }^{1}$, Zhijia $\mathrm{Li}^{1}$, Dequan Zeng ${ }^{1}$ \\ ${ }^{1}$ Dazhou Academy of Agricultural Sciences, Dazhou, Sichuan, China
}

\begin{abstract}
Selenium-enriched yeast has the physiological functions of improving immunity, anti-oxidation, liver protection, lipid-lowering and anti-cancer. Currently, it is widely used as an organic selenium supplement to prevent diseases of livestock and poultry and aquatic animals, and increase the rate of littering and egg production rate of poultry, reduce the mortality of fish and other aquatic animals, and promote the growth of livestock and poultry. This article reviews the acute toxicity, genotoxicity and subchronic toxicity of selenium-enriched yeast, in order to provide a reference for the safe application of selenium-enriched yeast.
\end{abstract}

\section{Introduction}

In 1817, Swedish scientist Berzelius discovered selenium. Selenium is a trace element necessary for human and animal body growth and development. It is an essential component of the body's antioxidant system. It is mainly involved in the reduction of hydrogen peroxide catalyzed by glutathione peroxidase (GSH-Px). Thereby reducing the generation of active oxygen and free radicals. In addition, selenium also has important physiological functions such as improving animal immune function, anti-tumor, reducing heavy metal toxicity, resisting radiation damage, and regulating the balance of thyroid metabolism ${ }^{[1-5]}$. However, animals cannot synthesize selenium by themselves and must consume food. Insufficient intake will cause various diseases, such as Keshan disease, Kashin-Beck disease, macular retinopathy, cognitive impairment, and decreased immunity. Selenium is abundant in China but unevenly distributed, and the phenomenon of selenium deficiency is widespread. Strengthening selenium through food has become one of the current research hotspots. There are two main forms of selenium supplements, inorganic selenium (mainly sodium selenite) and organic selenium. However, inorganic selenium has high toxicity, low bioavailability, and potential pollution to the environment, so some countries The use of inorganic selenium (sodium selenite) as a selenium supplement has been restricted. Organic selenium mainly exists in the form of selenomethionine or selenium polysaccharide. It has little toxic and side effects, is easy to absorb, and has high bioavailability. Therefore, it has a large application space as a food additive. Currently, selenium-enriched yeast is mostly used.

Selenium-enriched yeast uses bioengineering technology to combine inorganic selenium with macromolecular substances such as proteins and sugars in yeast cells to obtain organic selenium, which has physiological functions such as improving immunity, antioxidant, liver protection, lipid-lowering and anticancer $^{[6-8]}$. Different regions have different needs for selenium, so when supplementing selenium, it should be determined according to different regions and different ages. According to Chinese nutrition studies, the daily recommended amount of selenium intake for people of different ages is shown in Table 1. But when its intake is too high, it will also produce toxicity. The toxic mechanism of selenium currently mainly has the following three views: The first is the active oxygen radical theory, this theory believes that the hydrogen selenide generated by the reaction of sodium selenite with glutathione, reacts with oxygen to produce superoxide anions With other forms of active oxygen, the free radicals generated by the peroxidation of selenium can affect the structure of biomolecules, especially phospholipids, leading to the peroxidation of phospholipids, thereby affecting the function of biofilms; the second is the theory of selenium toxicity hypothesis It is believed that the toxicity of selenium may depend on the rate of renal excretion of selenium and the rate of methylation in the body; the third is that the toxicity of selenium may be related to the loss of Sadenosylmethionine. This article reviews the acute toxicity, genotoxicity and subchronic toxicity of selenium-enriched yeast, and aims to provide a basis for the wide application of selenium-enriched yeast.

Table1. The recommend amount of selenium for different age of people

\begin{tabular}{ccc}
\hline Group & Age & Selenium(ug) \\
\hline baby & $0 \sim 0.5$ & $10 \sim 40$ \\
children & $0.5 \sim 1$ & $20 \sim 60$ \\
& $1 \sim 3$ & $20 \sim 80$
\end{tabular}




\begin{tabular}{ccc} 
& $4 \sim 6$ & $30 \sim 120$ \\
teenager & $11 \sim 18$ & $50 \sim 200$ \\
& adult & $50 \sim 250$ \\
\hline
\end{tabular}

\section{Acute toxicity test}

Acute toxicity test is the most basic content in toxicology test, and it is the first step in the study and understanding of chemical toxicity. Acute toxicity refers to the poisoning reaction produced by the body in a short time (up to $14 \mathrm{~d}$ ) after a single exposure or multiple exposures to chemicals within $24 \mathrm{~h}$, and understand the toxicity strength, nature and possibility of the test substance. The target organ is to determine the lethal dose $\left(\mathrm{LD}_{50}\right)$. Yu Yingjie ${ }^{[9]}$ and others have took 77 Kunming mice as the test object, used simplified Kou's method, and gave them fasting intragastric administration respectively at different doses to observe the death of the tested mice and studied the acute toxicity of selenium-enriched yeast. The LD50 of seleniumenriched yeast is $26.38 \mathrm{~g} / \mathrm{kg}$, greater than $20 \mathrm{~g} / \mathrm{kg}$, which is actually non-toxic. Yao Wenhuan ${ }^{[10]}$ and others have studied the acute oral toxicity test in mice, and the results are consistent with that of $\mathrm{Yu}$ Yingjie. Seleniumenriched yeast is actually nontoxic. Guo Jie ${ }^{[11]}$ and others further studied the toxicological safety of seleniumenriched yeast of Ganoderma lucidum spore powder. The results showed that selenium-enriched yeast of Ganoderma spore powder was also a non-toxic substance.

\section{Genotoxicity (mutagenicity) test}

The genotoxicity test is mainly to detect the mutagenicity of the test substance, and through such tests to qualitatively indicate whether the test substance has a mutagenic effect or a potential carcinogenic effect. When a mutation occurs in a somatic cell, the body will become cancerous, and the mouse white bone marrow micronucleus test is generally used; when the mutation occurs in a germ cell, it will affect the safety of the next generation, and the white mouse sperm aberration test is generally used.

\subsection{Micronucleus test of mouse bone marrow}

Micronucleus is a chromatin body that remains in the cytoplasm of a fragment of a chromosome or an entire chromosome that does not fit into the nucleus according to normal procedures during cell division. The purpose of the micronucleus test is to check whether the test substance can produce micronucleus in the test animal. The main objects of the test are the DNA cutting agent and aneuploid mutagen. Yu Yingjie ${ }^{[9]}$ and others have took 50 Kunming mice as test subjects, and set up three test groups with different doses, a cyclophosphamide positive control group and a distilled water negative control group, which were gavaged at one time and sacrificed after 24 hours to obtain a chest. Bone marrow fluid, smear, staining, microscopic examination of polychromatic erythrocytes, the results showed that compared with the negative control group, the incidence of micronucleus in each test group was not significant, the result was negative. The research results of Yao Wenhuan $^{[10]}$, Guo Jie ${ }^{[11]}$ and others are all negative.

\subsection{Sperm aberration test in mice}

Sperm aberration refers to abnormal changes in sperm morphology, which can be highly inherited to the next generation. The sperm morphology is determined by common chromosomes and sex chromosomes. $\mathrm{Yu}$ Yingjie ${ }^{[9]}$ and others have took 25 Kunming mice to test the teratogenicity of different doses of selenium-enriched yeast on mouse sperm. The results showed that selenium-enriched yeast had no teratogenicity on mouse sperm. The research results of Yao Wenhuan ${ }^{[10]}$ and Guo $\mathrm{Jie}^{[11]}$ are consistent with this, indicating that seleniumenriched yeast does not show genotoxic effects.

\section{Subchronic toxicity test (30d feeding test)}

The sub-chronic toxicity test is also called the 30-day feeding test, which is mainly to study the relationship between the sub-chronic toxicity dose and the response (effect) of the test substance, to determine the minimum dose of the harmful effect that can be seen by the naked eye and the maximum dose of the harmful effect that cannot be observed by the naked eye; Toxicological effects and toxicity-generating organs of the subchronic toxicity of the test substance; to study the reversibility of the subchronic toxicity damage of the test substance; to provide new information for the toxicological properties found or not found in the previous test, and to test the toxicity of the test substance Provide the basis for the mechanism of action and extrapolation of research to people. Yu Yingjie ${ }^{[9]}$ and others have took 60 Kunming mice as the test object and studied the accumulation toxicity of selenium-enriched yeast by periodically increasing the dose. The results showed that the accumulation coefficient of selenium-enriched yeast was 4.263 , which was moderate accumulation. The results of the 30-day feeding test by Yao Wenhuan ${ }^{[10]}$ and others showed that the animals in each experimental group grew well. The blood routine and blood biochemical indicators were within the normal range of this laboratory, and no meaningful pathological changes were seen. Zhao Kangtao ${ }^{[12]}$ and others studied the safety of the compatible product of selenium-enriched yeast and taurine. The results showed that during the test period, the growth activities of rats were normal. Within the normal range of this laboratory, no toxic reactions were seen.

With people's in-depth understanding of the biological function of selenium, selenium has been widely used in the prevention of diseases of livestock and poultry and aquatic animals, increase the rate of littering and egg production of livestock, reduce the mortality of fish and other aquatic animals, and promote The growth of livestock and poultry. Selenium-enriched yeast has been widely used because it can solve the toxicity problem of inorganic selenium to the body and 
improve the absorption and utilization rate of selenium by the body. However, due to the small buffer range between the nutritional requirements and poisoning of selenium, special attention should be paid to avoid excessive use of selenium fortifiers during the development and production of selenium-rich products. The propaganda and guidance of the agent urges the producers to abide by the relevant standards. At the same time, it is also necessary to strengthen the sampling and risk assessment of selenium-rich animal products to ensure the safety of selenium-rich animal products.

\section{References}

1. ZHANG Ling, ZHANG Yan-xin. The Physiological Function of Selenium and Its Application in Fruits Production[J]. Northern Fruits, 2018.

2. Liangliang D. The Physiological Function of Selenium and the Development of Selenium-rich Health Food[J]. modern food, 2018.

3. Min G X, Fang N J, Zheng F, et al. On the forms of selenium in plant and mechanisms of physiological role of selenium $[\mathrm{J}]$. journal of agricultural university of hebei, 2003.

4. Da-Hui L, Wen-Bing Z, Duan-Wei Z, et al. General survey on physiological function of selenium in plant $[\mathrm{J}]$. journal of mountain agriculture and biology, 2005.

5. Xiumei L I, Tao X U, Guohua S, et al. Effects of Dietary Selenium-Enriched Yeast Levels on Important Physiological Enzymes and Enrichment of Selenium in Sea Cucumber (Apostichopus japonicus)[J]. progress in fishery sciences, 2017.

6. Zeng R, Farooq M U, Zhang G, et al. Dissecting the Potential of Selenoproteins Extracted from Selenium-Enriched Rice on Physiological, Biochemical and Anti-Ageing Effects In Vivo[J]. Biological trace element research, 2019.

7. Zhonghao Z, Qiuyan W, Chen C, et al. Comparison of the effects of selenomethionine and seleniumenriched yeast in the triple-transgenic mouse model of Alzheimer's disease[J]. Food \& Function, 2018.

8. A. Sobolev, B. Gutyj, N. Grynevych, et al. Enrichment of meat products with selenium by its introduction to mixed feed compounds for birds $[\mathrm{J}]$. 2017.

9. $\mathrm{Yu}$ Yingjie, $\mathrm{Xu}$ Xiaojing, Li Yuanliang, Huang Kehe, et al. Experimental study on the safety of selenium-enriched yeast [J]. Animal Husbandry and Veterinary Medicine, 2006,38 (9): 38-40.

10. Yao Wenhuan, Xie Wei, Yang Fei, Yan Yan, Guo Jie, Han Xiaoying, study on acute, subacute and genotoxicity of selenium-enriched yeast $[\mathrm{J}]$. Chinese Journal of Sanitary Inspection, 2012.

11. Guo Jie, Xie Wei, Yan Yan, Lu Lianhua, Yao Wenhuan, et al. Toxicological safety evaluation of selenium-enriched yeast preparation of Ganoderma lucidum spore powder [J]. Journal of Toxicology, 2017, 31 (6) 491-494.
12. Zhao Kangtao, Ai Fuqin, Zheng Lihong, Chen Xiujin, Lin Jian, Safety study on the compatibility of selenium-enriched yeast and taurine [J]. Strait Journal of Preventive Medicine, 2019. 